\title{
HRCT Chest Evaluation of COVID-19 Patients: Experience in Combined Military Hospital Dhaka, Bangladesh
}

\author{
SZ ALAM $^{\mathrm{a}}$, SMAA MUID $^{\mathrm{b}}$, AAKHTER $^{\mathrm{c}}$, AKMS RAHMAN $^{\mathrm{d}}$, MA EMRAN $^{\mathrm{e}}$, MTA MOSTAKIM $^{\mathrm{f}}$
}

\begin{abstract}
:
Background: HRCT Chest is an important tool in both diagnosis and management of COVID-19 patient, as well as it is an important complement to the reverse-transcription polymerase chain reaction (RT-PCR) tests.

Purpose: The purpose of this study is to assess different patterns of manifestation in HRCT chest in COVID-19 infection \& to grade the severity by observing a sample of 128 after the symptoms began.

Method: From 11 April 2020 to 27 May 2020, 128 patients who were admitted in Combined Military Hospital Dhaka and underwent both HRCT chest and RT-PCR for COVID19 were included. Distribution and patterns of pulmonary lesions like ground glass opacity (GGO), consolidation, reverse halo sign, crazy paving, thickened vascular marking, lymphadenopathy and pleural effusion were evaluated.
\end{abstract}

Result: Total 128 patients diagnosed (RT-PCR Positive) with COVID-19 were included. Among them, 112(87.5\%) patients had fever, and $58(45.31 \%)$ patients had fatigability. The most frequent CT abnormality was ground glass opacity

Introduction:

In December 2019, an outbreak of pneumonia of unknown etiology was reported in Wuhan, Hubei province, China. The pathogen was a novel corona virus named severe acute respiratory syndrome corona virus 2 (SARS-CoV-2) by WHO and the disease caused by

a. Professor (Brig Gen) Dr. Syed Zoherul Alam, Advisor and Head of the Department, Department of Radiology \& imaging, CMH DHAKA

b. Professor (Brig Gen) Dr. SMA Al Muid, Advisor specialist in Radiology \& Imaging, Department of Radiology \& Imaging, CMH DHAKA

c. Col (Dr.) Afroza Akhter, Classified specialist in Radiology \& Imaging, Department of Radiology \& Imaging, $\mathrm{CMH}$ DHAKA

d. Lt Col (Dr.) AKM Sharifur Rahman, Classified specialist in Radiology \& Imaging, Department of Radiology \& Imaging, CMH DHAKA

e. Major (Dr.) Md. Al Emran, Graded Specialist in Radiology \& Imaging, Armed Forces Medical Institute, Dhaka

f. Major (Dr.) Md. Tarif Al Mostakim, Graded Specialist in Radiology \& Imaging, Armed Forces Medical Institute, Dhaka

Address of the Correspondence: Professor (Brig Gen) Dr. Syed Zoherul Alam U-11, Uttoron, Aziz palli, Dhaka Cantonment Mobile: 01769014856,01711703515, E-mail: zahir639@yahoo.com in 123(96.09\%) cases. Amongst them 81(63.28\%) cases had GGO plus consolidation and ground glass opacity alone were $42(32.81 \%)$ cases. Crazy-paving pattern was in 65(50.78\%) cases. Most patients had multiple lesions and involved all the 5 lobes in 96(75.00\%) cases. The lesions were mostly peripheral (123,96.09\%) and posterior $(103,80.47 \%)$ and in $65(50.78 \%)$ cases the distribution were diffuse but predominantly peripheral. Most commonly involved lobe is right lower lobe (120,93.75\%) and left lower lobe(117,91.41\%).

Conclusion: HRCT chest can play an important role in the early diagnosis and prompt management of this global health emergency.

Key words: $R T-P C R=$ Reverse transcription polymerase chain reaction, $H R C T=H i g h$ resolution computed tomography, COVID-19=corona virus disease 2019, GGO=ground glass opacity, $\mathrm{WHO}=$ World Health Organization, $\mathrm{CMH}=$ Combined Military Hospital.

(J Bangladesh Coll Phys Surg 2020; 38: 21-28) DOI: https://doi.org/10.3329/jbcps.v38i0.47441

SARS-CoV-2 was termed as corona virus disease 2019 (COVID-19). ${ }^{1}$ The World Health Organization (WHO) on March 11,2020, has declared the novel corona virus (COVID-19) outbreak a global pandemic. ${ }^{2}$

Until 27 May 2020, the number of confirmed cases in Bangladesh has risen to 36751 , of which 522 have died. ${ }^{3}$

This new corona virus spread from one person to another primarily through respiratory droplets generated when an infected person coughs or sneezes. ${ }^{4}$ Symptoms of infection include: fever, cough and shortness of breath. ${ }^{5}$ At present, the diagnosis of COVID-19 pneumonia is based on clinical symptoms, contact history of epidemic area, imaging diagnosis and nucleic acid detection. ${ }^{1}$ RTPCR is believed to be highly specific, but with sensitivity reported as low as $60-70 \%$ and as high as $95-$ $97 \%$. Thus, false negatives are a real clinical problem. ${ }^{4,6}$

High resolution Computed tomography (HRCT) of the chest is increasingly recognized as strong evidence for early diagnosis, because the changes in chest imaging sometimes may be earlier than clinical symptoms and thus HRCT scan play an early warning role in the diagnosis of COVID-19. ${ }^{7}$ Recent studies have demonstrated that HRCT can play a critical role in the 
early identification of pneumonia and help in accurate diagnosis as HRCT has high sensitivity of $97 \%$ in diagnosing COVID-19. ${ }^{8}$

AIM:

1. To assess different patterns of manifestation in HRCT chest.

2. To grade the severity of lung involvement in COVID19 infection.

\section{Materials and Methods:}

\section{Study design}

A descriptive, cross sectional study design was used to examine RT-PCR positive COVID-19 patients.

\section{Place and duration of the study}

This study was done in Radiology \& Imaging Department, Combined Military Hospital Dhaka. Data were collected in the period spanned from 11 April 2020 up to 27 May 2020. Verbal consent was obtained from all potential participants/guardians. The aims and benefits of the present study were explained to all the participants in details. Medical history of all study subjects were thoroughly reviewed directly from participants themselves and from case sheet.

\section{Study population}

Total 128 patients were selected for study. Confirmed cases of COVID-19 pneumonia were admitted in CMH Dhaka and all the patients underwent HRCT chest. One patient underwent serial follow up HRCT chest for three times.

\section{Image evaluation}

All HRCT chest images were evaluated by two radiologists with a minimum experience of 10 years and all patients were evaluated to identify any change occurred within lung parenchyma. Patterns and distributions of lung involvement were evaluated. Visual quantitative evaluation for each of the five lung lobes were also assessed for degree of involvement and classified as none (0\%), minimal (1-25\%), mild (26-50\%), moderate (51-75\%) and severe (76-100\%). The total severity score was reached by summing the score of all five lobes (range of total severity score is $0-20) .{ }^{9}$

\section{Data Analyses}

The data were collected from CT reports and then stored in data sheet which prepared specially for this task. Data analyzed by using Microsoft excel and statistical package for the social sciences (SPSS) IBM version 25.

\section{Machine and parameters used}

For all scanning techniques (axial, coronal and/or sagittal), American, General Electric (GE) Hi- speed 128 slice multidetector CT scanner was used to obtain the HRCT chest.
The scanning parameter was $120 \mathrm{kV}, 200 \mathrm{mAs}$; matrix was $512 \times 512$; scanning time was $0.55 \mathrm{~s} /$ circuit; collimator was $0.625 \mathrm{~m}$; pitch was 0.89 , FOV $360 \mathrm{~mm}$; scanning thickness was $0.625 \mathrm{~mm}$; reconstruction algorithm: high spatial frequency, window: lung window. The scan ranged from the thoracic entrance to the angle plane of the bilateral rib. Level of inspiration: full inspiration. Proper protections of all the radiology staffs were taken. Out of three CT scanner machines in Radiology and Imaging department of CMH Dhaka, one scanner was dedicated for COVID19 patients.

\section{Results:}

The demographic data are shown in Table I. A total of 128 confirmed patients of COVID-19 including 47(36.72\%) female and $81(63.28 \%)$ male, their age ranging from 24 to 83 years with a mean age of $49.97 \pm 13.6($ SD). Highest number of the patients (43 patients, $33.59 \%$ ) were in age group 51-60 years and no patient was found below 20 years during our study. 28(21.88\%) patients were above the 60 years age group. There were past history of smoking, bronchial asthma and COPD in 22(17.19\%), $5(3.91 \%)$ and $6(4.69 \%)$ patients respectively.

Most of the patients presented with fever $112(87.50 \%)$ and the least common symptom was runny nose $9(7.03 \%)$. The patients were also presented with sore throat $(55,42.97 \%)$, dry cough $(22,17.19 \%)$, headache $(33,25.78 \%)$, fatigue $(58,45.31 \%)$, shortness of breath $(63,49.21 \%)$ and few were asymptomatic $(5,3.91 \%)$.

Among 128 patients who were RT-PCR positive, we have found positive HRCT findings in 123 cases. In 5 cases the lung findings were normal. Thus in our study we have found that HRCT has a sensitivity of $96.09 \%$. The most frequent CT abnormalities observed were ground glass opacity in $123(96.09 \%)$ cases. Among them $81(63.28 \%)$ cases had GGO plus consolidations and ground glass opacity alone were $42(32.81 \%)$ cases. Crazypaving pattern was seen in $65(50.78 \%)$ cases. Most of the lesions were multiple and involved all the 5 lobes in 96(75.0\%) cases. The lesions were distributed mostly at the periphery $(123,96.09 \%)$ and posterior $(103,80.47 \%)$ region. In $65(50.78 \%)$ cases the distribution were diffuse but predominantly peripheral. Most commonly involved lobe was right lower lobe $(120,93.75 \%)$ followed by left lower lobe $(117,91.41 \%)$. Among other findings reverse halo sign $(16,12.50 \%)$, sub-pleural band $(9,7.03 \%)$, thickened vessels $(13,10.16 \%)$, enlarged lymph nodes $(5,3.91 \%)$ were also observed as shown in Table II. No single case of pleural effusion was found in our study. Thus the most common consistent finding was GGO in peripheral and posterior distribution. There was no significant gender differentiation of HRCT chest findings. 
There were 5(3.91\%) cases with unilateral and $118(92.19 \%)$ cases with bilateral lung involvement. Out of 78 positive HRCT findings, all 5 lobes were involved in 96(75.0\%) cases, 4 lobes were involved in 13(10.16\%) cases, 3 lobes were involved in $8(6.25 \%)$ cases, 2 lobes were involved in $3(2.34 \%)$ cases and only one lobe was involved in $3(2.34 \%)$ cases. No abnormal finding in HRCT chest was seen in 5 (3.91\%) cases who were RT-PCR positive. Right lower lobe was involved in most of the cases $(120,93.75 \%)$ and least common involvement was in right middle lobe $(96,75.0 \%)$ as shown in Table II.

Total severity score was categorized into 5 groups $(0,1-$ 5, 6-10, 11-15, 16-20). Most of the patients (38,29.69\%) were in total severity score ranging from 1-5. Among others sequential distribution of total severity score ranges are 6-10 $(33,25.78 \%), 11-15(27,21.09 \%), 16-20$ $(25,19.53 \%)$ and severity score 0 was of $5(3.91 \%)$ patients. In case of total percentage of lung involvement maximum patients $(38,29.69 \%)$ were in the range of 1 $25 \%$ and maximum lung involvement (76-100\%) was seen in $25(19.53 \%)$ cases as shown in Table III.

Table-I

\begin{tabular}{lcc}
\multicolumn{2}{c}{ Characteristics of enrolled patients } & $(n=128)$ \\
Characteristics & Number & Percentage \\
\hline Age (years) & & \\
$<20$ & 0 & 0 \\
$21-30$ & 8 & 6.25 \\
$31-40$ & 22 & 17.19 \\
$41-50$ & 27 & 21.09 \\
$51-60$ & 43 & 33.59 \\
$>60$ & 28 & 21.88 \\
Sex distribution & & \\
Male & 81 & 63.28 \\
Female & 47 & 36.72 \\
Personal history & & \\
Smoker & 22 & 17.19 \\
Bronchial asthma & 5 & 3.91 \\
COPD & 6 & 4.69 \\
Clinical features & & \\
Fever & 112 & 87.50 \\
Sore throat & 55 & 42.97 \\
Dry cough & 22 & 17.19 \\
Headache & 33 & 25.78 \\
Runny nose & 9 & 7.03 \\
Fatigue & 58 & 45.31 \\
Shortness of breath & 63 & 49.21 \\
\hline
\end{tabular}

Note: Mean age is 49.97 years ( $\mathrm{SD} \pm 13.6$ ) with a range of 24-83 years.

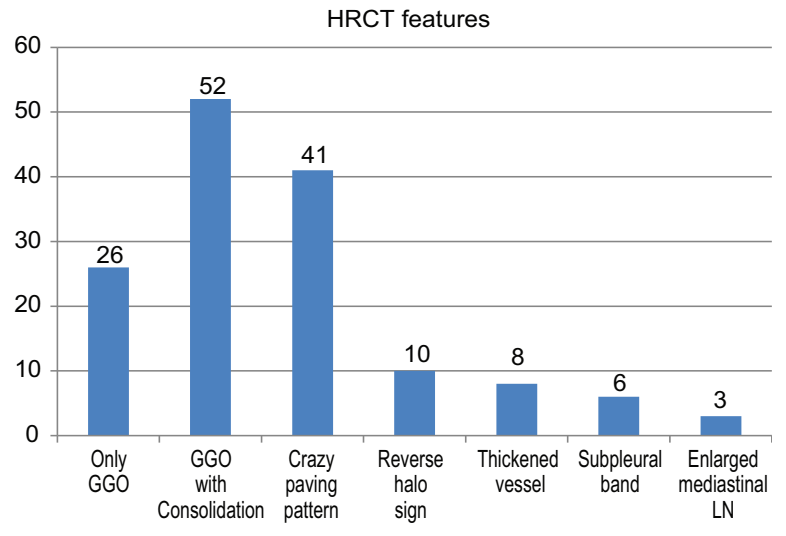

Fig.-1: Distribution of cases according to HRCT chest features.

Table-II

Findings of HRCT chest along with lobar involvement and distribution

Findings Number of patients

\section{HRCT Patterns}

Ground glass opacity(GGO)

$42(32.81 \%)$

GGO with Consolidation

$81(63.28 \%)$

Crazy paving pattern

$65(50.78 \%)$

Reverse halo sign

$16(12.50 \%)$

Thickened vessels

$13(10.16 \%)$

Sub-pleural band

$9(7.03 \%)$

Pleural effusion

0

Enlarged mediastinal nodes

$5(3.91 \%)$

Number of lobes involved

$5(3.91 \%)$

$3(2.34 \%)$

$3(2.34 \%)$

$8(6.25 \%)$

$13(10.16 \%)$

$96(75.0 \%)$

Frequency of lobar involvement

Right upper lobe

$112(87.50 \%)$

Right middle lobe

$96(75.0 \%)$

Right lower lobe

$120(93.75 \%)$

Left upper lobe

$110(85.94 \%)$

Left lower lobe

$117(91.41 \%)$

Distribution of involvement

Central

0

Peripheral

$58(45.31 \%)$

Diffuse predominantly peripheral

$65(50.78 \%)$

Note: Bilateral involvement was in 119(92.97\%) cases. 


\section{Table-III}

\section{Severity score and lung involvement}

Findings

Number of patients

\section{Distribution on the basis of total severity score}

0 $5(3.91 \%)$

$1-5$

$38(29.69 \%)$

6-10

$33(25.78 \%)$

$11-15$

$27(21.09 \%)$

16-20

$25(19.53 \%)$

\section{Total percentage of lung involvement}

$0 \%$

$$
5(3.91 \%)
$$

$1-25 \%$

$38(29.69 \%)$

$26-50 \%$

$33(25.78 \%)$

$51-75 \%$

$27(21.09 \%)$

$76-100 \%$

$25(19.53 \%)$

Total

128

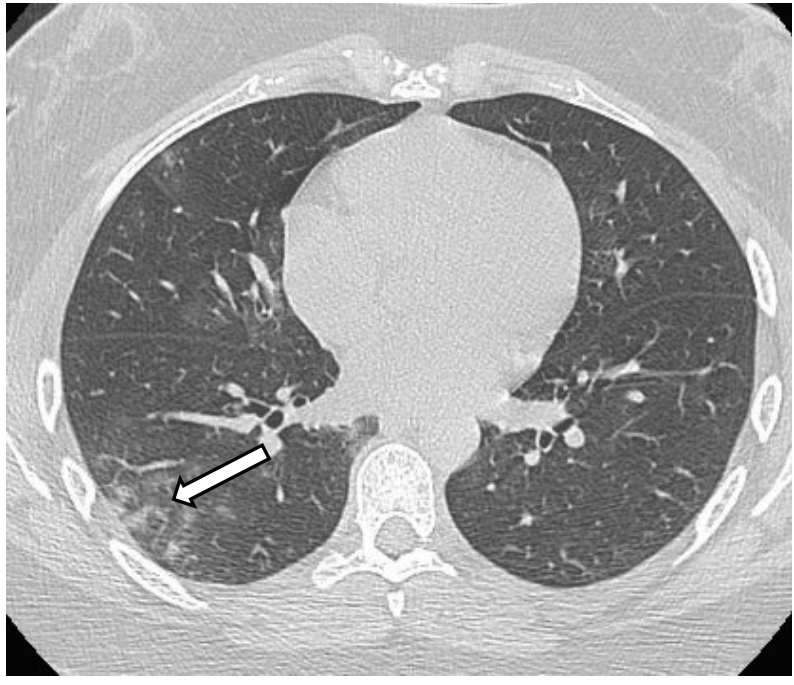

(a)

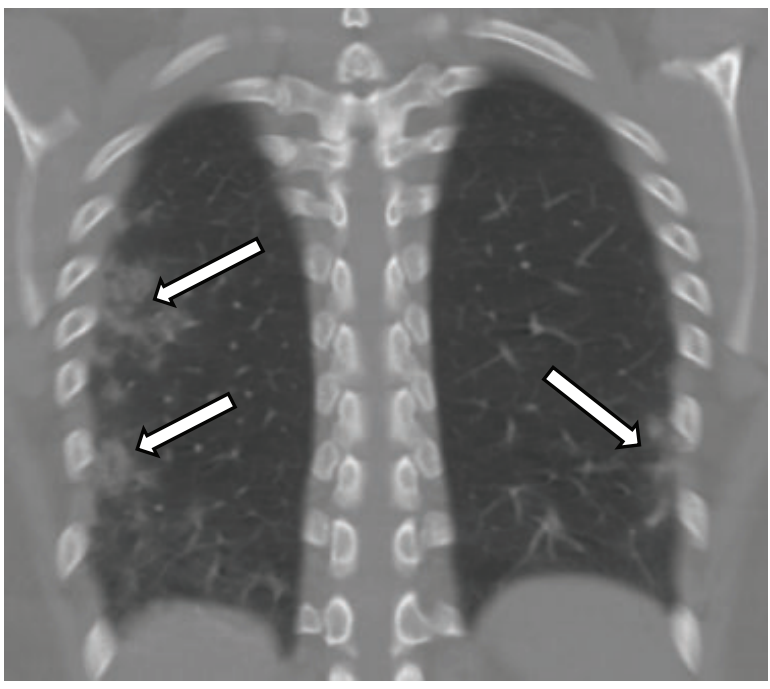

(b)

Fig.-2: HRCT chest images (done on 12 April 2020) of a 37 years old lady with fever for 5 days. Positive result for RT-PCR assay for COVID-19 using a nasal swab was obtained on 10 April 2020. Chest axial HRCT image (a) showing multiple foci of GGOs (white arrows) distributed peripherally involving posterior and lateral segments of right lower lobe. Reconstructed coronal image (b) shows bilateral distribution of GGOs (white arrows). 


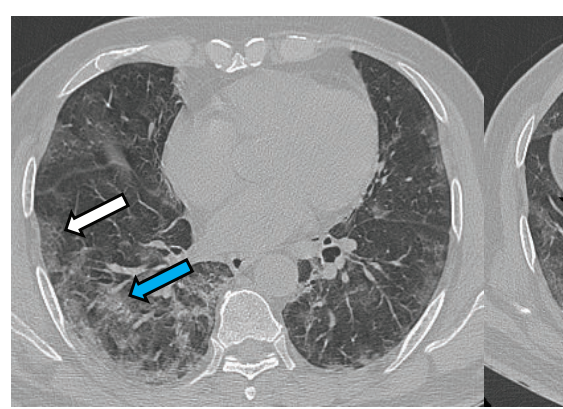

(a)

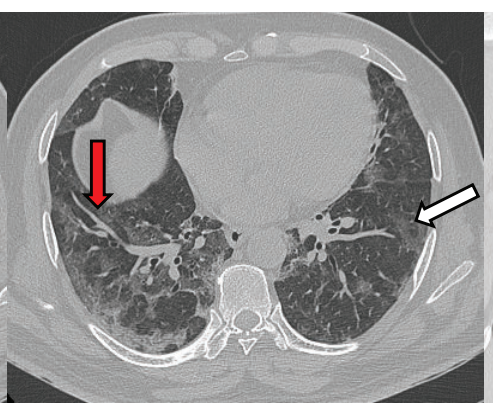

(b)

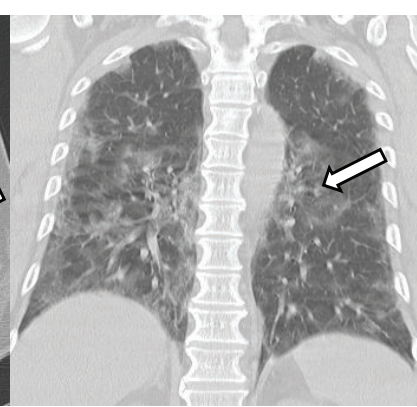

(c)

Fig.-3: HRCT chest image of a 58 years old male done after 8 days of onset of symptoms. Multifocal areas of GGOs (white arrow), involving all the visible lobes of both lungs in diffuse but predominantly peripheral and posterior distribution, seen in axial HRCT images ( $a \& b)$ and in reformatted coronal image(c). Small areas of soft tissue densities with air bronchogram (blue arrow) are observed. Thickened vessel is also seen (red arrow).

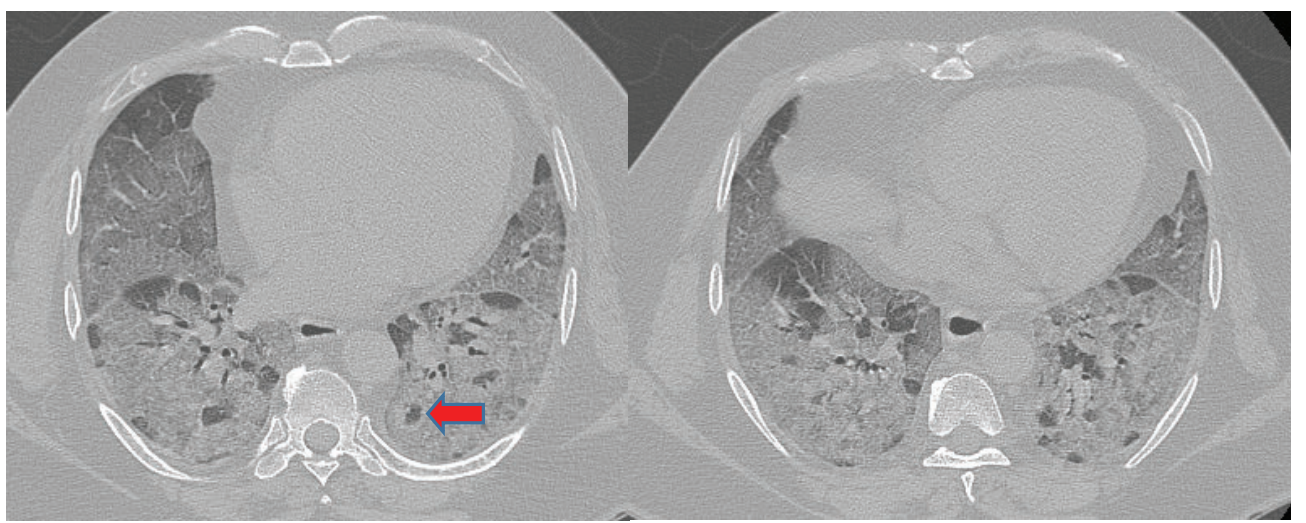

(a)

(b)

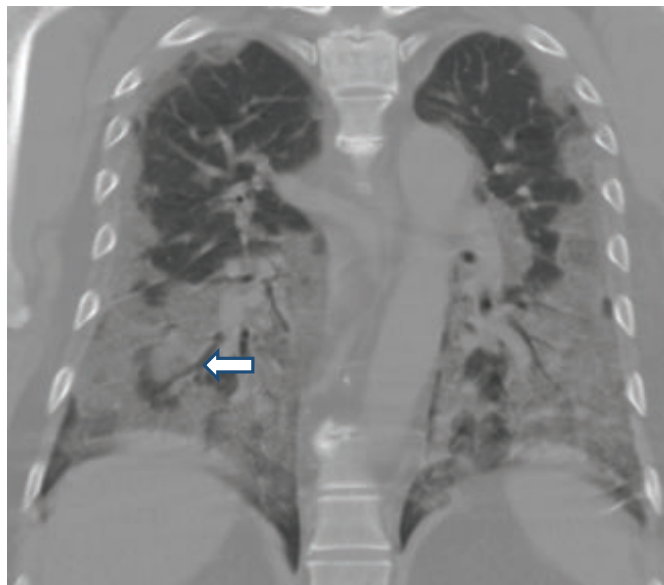

(c)

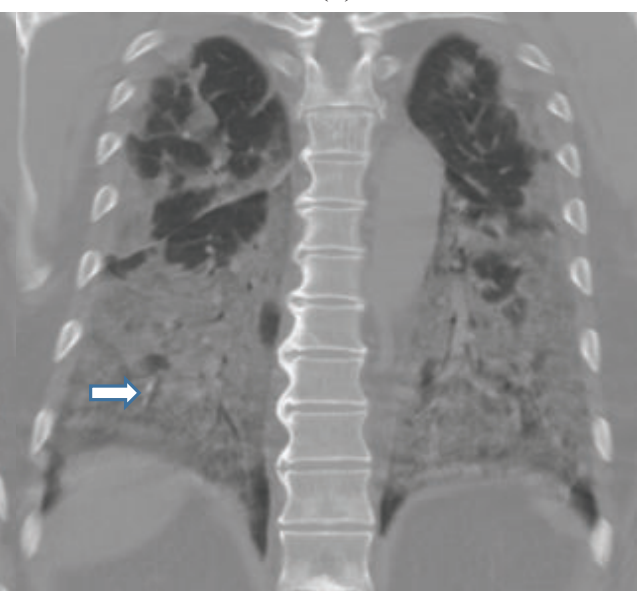

(d)

Fig.-4: HRCT chest image of a 65 years old male 11 days after the onset of symptom. Axial ( $a \& b)$ and reformatted coronal $(c \& d)$ images show diffuse GGOs with thickened intra and interlobular septae, producing crazy paving pattern involving all the visible segments of both lungs with predominant involvement at basal segments of lower lobes, mostly in peripheral distribution. Soft tissue density areas with air bronchogram (white arrows) are also seen. Reverse halo sign is also visible (red arrow). His severity score was 18 out of 20 and the patient died 5 days after doing this HRCT. 

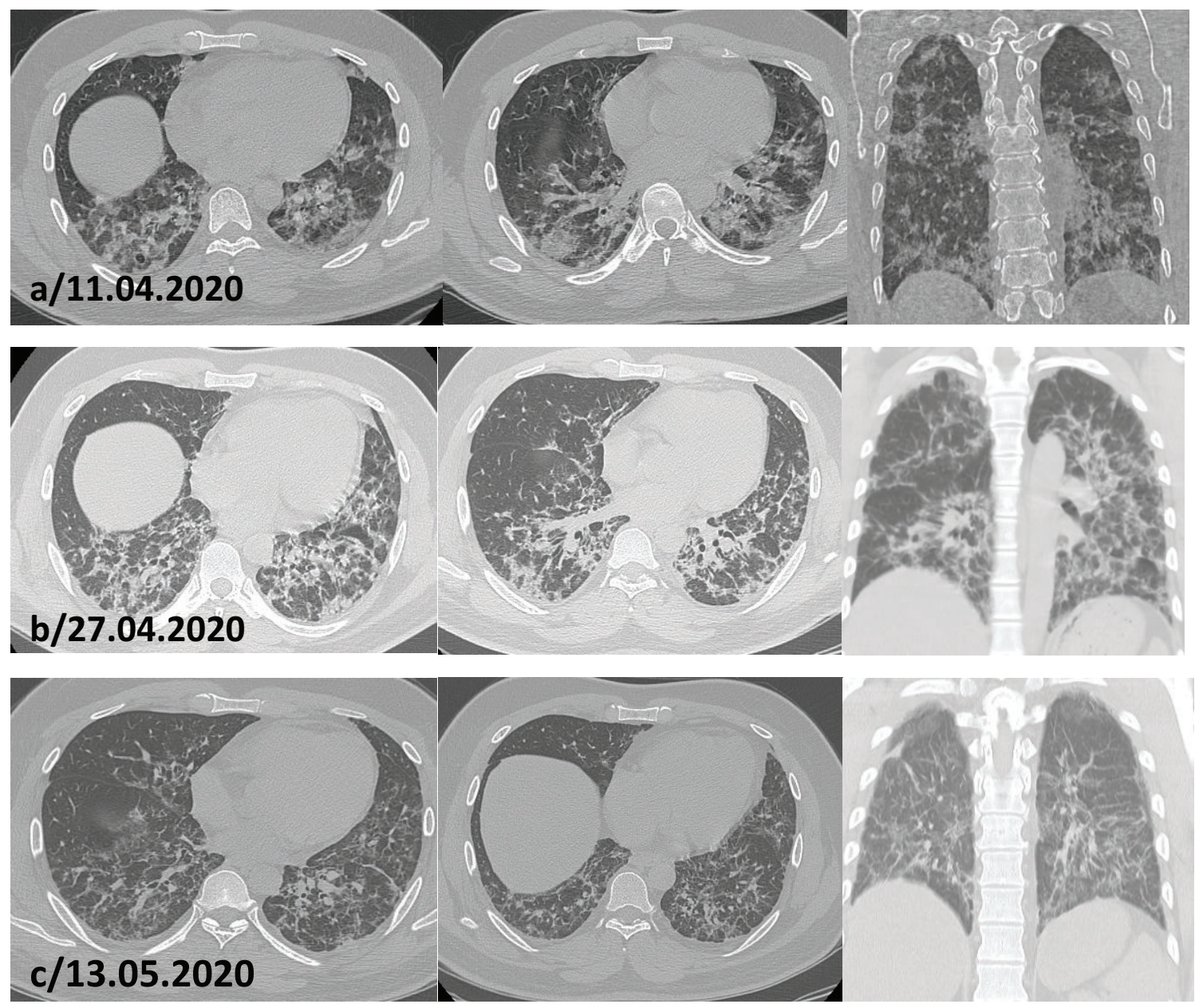

Fig.-5: Serial HRCT scan of chest. Positive result of RT-PCR assay for COVID-19 using swab sample was obtained on 7th April (row a). Negative RT-PCR was obtained on 27th April (row b). Follow up HRCT scan after discharge from hospital (row c). In first scan there was diffuse ground glass opacities and soft tissue density areas with air bronchogram are seen, involving all the visible lobes of both lungs and had total severity score of 16/20. In second scan, areas of distribution were almost same but some reduction of GGOs. At the same time new appearance of some fibrotic striations with the total severity score of 15/20. It denotes minimal regression of the progressive phase as well as gradual development of healing stage. The 3rd scan was done 41 days after the onset of symptom and 17 days after the declaration of COVID-19 negative. There was significant reduction of GGOs but fibrotic striations became more prominent. It denotes the patient is gradually recovering from the disease. In all the three scans, there were no lymphadenopathy or pleural effusion.

\section{Discussion:}

The present study was conducted on 128 confirmed cases of COVID-19(RT-PCR positive) in Combined Military Hospital Dhaka. In our study we have found that HRCT chest has a sensitivity of $96.09 \%$ in diagnosing COVID-19 pneumonia which is almost very similar to other studies. Ai $\mathrm{T}$ et al in a study to find out the correlation of chest CT and RT-PCR testing in corona virus disease 2019 in China concluded that
HRCT has 97\% sensitivity in diagnosing COVID- $19 .{ }^{8}$ Wen $\mathrm{Z}$ et al found that HRCT chest has a sensitivity of $93 \% .{ }^{10}$ Thus HRCT images with $96.09 \%$ sensitivity can play an important role in the rapid diagnosis and evaluation of COVID-19 and can be used as a standard method in timely management of patients.

A greater number of male patient $(81,63.28 \%)$ were found than that of female $(47,36.72 \%)$, which was similar to previous study done by Chen $\mathrm{N}$ et al. ${ }^{11}$ The reduced 
susceptibility of females to viral infections might be attributed to the protection from $\mathrm{X}$ chromosome and sex hormones, which play an important role in innate and adaptive immunity. ${ }^{12}$ Moreover as it is a military hospital where most of the employed persons are male, it may be another cause of higher number of male patient in our study.

The most common suggestive finding was ground glass opacity (Figure $2 \& 3$ ) in peripheral $(123,96.09 \%)$ and posterior distribution $(103,80.47 \%)$. The prevalence of pure GGO in the study population was $42(32.81 \%)$, and GGO with consolidation (Figure 5) in the study population was $81(63.28 \%)$ which is almost similar to study done by Wen $\mathrm{Z}$ et al. ${ }^{10}$

Peripheral distribution was found in all cases, among them $58(45.31 \%)$ cases showed solely peripheral distribution and $65(50.78 \%)$ cases showed diffuse distribution with predominance in the periphery. In terms of distribution of the pulmonary opacities Wen $\mathrm{Z}$ et al ${ }^{10}$ found that out of 82 , in $63(77 \%$ ) cases the distribution of the lung opacities were peripheral, in $3(4 \%)$ cases the distribution were central, and in $16(20 \%)$ cases the distribution were both central and peripheral. Caruso D et al also showed in their study that out of 58 HRCT chest peripherally distributed cases were $52(89 \%$ with a CI of $81-98 \%){ }^{13}$

The crazy paving pattern finding in this study was $65(50.78 \%)$ out of 128 cases. Wu J et al ${ }^{15}$ in a study in china found crazy paving pattern in $76.9 \%$ cases. Wu J et $\mathrm{al}^{16}$ in another study showed that crazy paving pattern appear in $29 \%$ of cases. Thus crazy paving pattern has a wide range of appearance in different study.

The least common finding in our study was lymphadenopathy. In our case we found $5(3.91 \%)$ cases. Caruso D et al ${ }^{13}$ found no lymphadenopathy in their study while it was $2.7 \%$ in another study. ${ }^{14}$

In our study we found no single case of pleural effusion in HRCT chest finding. In other studies it is also an inconsistent finding. As in a study done by Wen $\mathrm{Z}$ et $\mathrm{al}^{10}$ they found only 1 case out of 103 COVID-19 positive patient. In another study it was found 2 out of 58 patients. ${ }^{13}$ Thus we can say that pleural effusion is a rare finding in case of COVID-19 patient.

Reverse halo sign (Figure 4), thickened vessel (Figure 3 ) and sub-pleural bands were less common finding in our study and it was found in 16(12.5\%), 13(10.16\%) and $9(7.03 \%)$ cases respectively.
In our study, maximum patients (38, 29.69\%) were in group of total severity score 1-5 and highest score 16-20 was found in 25(19.53\%) cases as shown in Table III. In our study, the calculation of the total percentage (\%) of lung parenchymal involvement was done by minimum 2 expert radiologists of minimum 10 years of experiences. The total percentage is calculated by summation of involved percentage of each 5 lobes divided by 5 . The result was the near possible percentage of total lung parenchymal involvement which was finally re-assessed visually as the pattern of involvement is not same in all patients as well as all lobes are not equal in size.

According to the degree of lung involvement evaluated by CT scoring summation of all the lobes of both lungs, the severity of lung involvement was comparatively less severe within 1 to 7 days of onset of symptoms than the onset of symptom in the range of 8 to 14 days stage. The findings are least severe after 14 days. In our study, we got $58(45.31 \%)$ cases in 1-7 days stage of which maximum $16(12.5 \%)$ cases were with minimal lung involvement and 5(3.91\%) cases were presented with severe involvement of lung parenchyma. In stage of 814 days, among total 59(46.09\%) patients, each of $18(14.06 \%)$ cases had moderate and severe involvement of lung parenchyma and minimum $8(6.25 \%)$ cases showed minimal involvement of lung parenchyma. In $>14$ days stage, there were $8(6.25 \%)$ patients with minimal lung involvement only (neither moderate nor severe). Zhou et al in a study on imaging features and evolution on CT in 100 COVID-19 pneumonia patients in Wuhan concluded with the inference that the early rapid progressive stage is 1 7 days from symptom onset, the advanced stage with peak levels of abnormalities on $\mathrm{CT}$ is $8 \sim 14$ days, and the abnormalities started to improve after 14 days. ${ }^{17}$

In one case there were series of HRCT scan done. The first scan (Figure 6a) was done on 12 April 2020 (8 days after the onset of symptom) where all the five lobe were involved with a severity score of 16 out of 20 with more severe involvement of both the lower lobes. Ground glass opacity predominantly in peripheral and posterior distribution was seen. Associated multifocal soft tissue density areas with air bronchogram were also marked in different segments. The 2nd scan(Figure 6b) was done 23 days after the onset of symptom on 27 April (he was also declared RT-PCR negative for COVID-19 on 27 April) which revealed almost the same areas of distribution but some reduction of GGOs with new appearance of few different patterns like appearance of crazy paving, 
fibrotic striations and thickened vessels. The severity score was 15 which denote minimal regression of disease. The 3rd scan (Figure 6c) was done on 13 May 2020 (41 days after the onset of symptom and 17 days after the declaration of COVID-19 negative). In this scan there were significant improvements of GGOs as well as marked fibrotic striations which indicate improvement of active stage followed by gradual development of stage of resolution. In all the three scans there were no lymphadenopathies or pleural effusion.

\section{Conclusions:}

In conclusion, the most common pattern of COVID-19 pneumonia on HRCT images are pure GGO, GGO with crazy paving or interlobular septal thickening and GGO with consolidation with prominent distribution in the posterior and peripheral part of the lungs.

HRCT Chest should be used for comprehensive evaluation, combined with the results of nucleic acid tests and the epidemiological data. In the context of typical clinical presentation and the exposure history to patients with COVID-19 pneumonia, in addition to the typical HRCT features we reported in the present study, the patients may be strongly suspicious of having SARS-CoV-2 infection, regardless of the RT-PCR results. HRCT imaging plays a vital role in the early clinical detection and diagnosis of COVID-19 pneumonia, and can be considered as clinical diagnostic modality.

Appearance and degree of lung involvement seen by HRCT chest plays an important role to modify the treatment plan at times in critically ill patient of COVID-19.

\section{References:}

1. Dai H, Zhang X, Xia J, Zhang T, Shang Y, Huang R, et al. High-resolution chest $\mathrm{CT}$ features and clinical characteristics of patients infected with COVID-19 in Jiangsu, China. International Journal of Infectious Diseases. 2020 Apr 6.

2. WHO Director-General's opening remarks at the media briefing on COVID19 -March 2020[Cited..]https:// www.who.int/dg/speeches/detail/who-director-generalsopening-remarks-at-the-media-briefing-on-covid-19

3. World Health Organization (May 27, 2020) Coronavirus disease 2019 (COVID-19). Situation report-83. Geneva, Switzerland: World Health Organization; 2020; https:// www.who.int/emergencies/diseases/novel-coronavirus2019/situation-reports.
4. Lei J, Li J, Li X, Qi X. CT imaging of the 2019 novel coronavirus (2019-nCoV) pneumonia. Radiology 2020 Jan 31

5. Li Q, Guan X, Wu P, Wang X, Zhou L, Tong Y, Ren R, Leung KS, Lau EH, Wong JY, Xing X. Early transmission dynamics in Wuhan, China, of novel coronavirus-infected pneumonia. New England Journal of Medicine. 2020 Jan 29.

6. Cheng Z, Lu Y, Cao Q, Qin L, Pan Z, Yan F, et al. Clinical features and chest CT manifestations of coronavirus disease 2019 (COVID-19) in a single-center study in Shanghai, China. American Journal of Roentgenology. 2020 Mar 14:1-6.

7. Wang H, Wei R, Rao G, Zhu J, Song B. Characteristic CT findings distinguishing 2019 novel coronavirus disease (COVID-19) from influenza pneumonia. European Radiology. 2020 Apr 22:1.

8. Ai T, Yang Z, Hou H, Zhan C, Chen C, Lv W, et al. Correlation of chest CT and RT-PCR testing in coronavirus disease 2019 (COVID-19) in China: A report of 1014 cases [published online ahead of print February 26, 2020]. Radiology.;10.

9. Bernheim A, Mei X, Huang M, Yang Y, Fayad ZA, Zhang $\mathrm{N}$, et al. Chest $\mathrm{CT}$ findings in coronavirus disease-19 (COVID-19): relationship to duration of infection. Radiology. 2020 Feb 20:200463.

10. Wen Z, Chi Y, Zhang L, Liu H, Du K, Li Z, et al. Coronavirus disease 2019: initial detection on chest $\mathrm{CT}$ in a retrospective multicenter study of 103 Chinese subjects. Radiology: Cardiothoracic Imaging. 2020 Apr 6;2(2):e200092.

11. Chen N, Zhou M, Dong X, Qu J, Gong F, Han Y, et al. Epidemiological and clinical characteristics of 99 cases of 2019 novel coronavirus pneumonia in Wuhan, China: a descriptive study. Lancet 2020;395:507-13.

12. Jaillon S, Berthenet K, Garlanda C. Sexual dimorphism in innate immunity. Clinical reviews in allergy \& immunology. 2017 Sep 30:1-4.13. Caruso D, Zerunian M, Polici M, Pucciarelli F, Polidori T, Rucci C, et al. Chest CT features of COVID-19 in Rome, Italy. Radiology. 2020 Apr 3:201237.

14. Bai HX, Hsieh B, Xiong Z, Halsey K, Choi JW, Tran TM, et al. Performance of radiologists in differentiating COVID19 from viral pneumonia on chest CT. Radiology. 2020 Mar 10:200823.

15. Wu J, Pan J, Teng D, Xu X, Feng J, Chen YC. Interpretation of CT signs of 2019 novel coronavirus (COVID-19) pneumonia. European Radiology. 2020 May 4:1-8.

16. Wu J, Wu X, Zeng W, Guo D, Fang Z, Chen L, et al. Chest CT findings in patients with coronavirus disease 2019 and its relationship with clinical features. Investigative radiology. 2020 May 1;55(5):257-61.

17. Zhou S, Zhu T, Wang Y, Xia L. Imaging features and evolution on CT in 100 COVID-19 pneumonia patients in Wuhan, China. European Radiology. 2020 May 4:1-9. 